\title{
Primary Biliary Cirrhosis-Specific Antimitochondrial Antibodies in Neonatal Haemochromatosis
}

\author{
Daniel S. Smyk, ${ }^{1}$ Maria G. Mytilinaiou, ${ }^{1,2}$ Tassos Grammatikopoulos, ${ }^{2}$ A. S. Knisely, ${ }^{1}$ \\ Giorgina Mieli-Vergani, ${ }^{1,2}$ Dimitrios P. Bogdanos, ${ }^{1,3}$ and Diego Vergani' \\ ${ }^{1}$ Institute of Liver Studies, GI and Nutrition Centre, King's College London School of Medicine at King's College Hospital, \\ Denmark Hill Campus, London SE5 9RS, UK \\ ${ }^{2}$ Paediatric Liver, GI and Nutrition Centre, King's College London School of Medicine at King's College Hospital, \\ Denmark Hill Campus, London SE5 9RS, UK \\ ${ }^{3}$ Department of Medicine, Faculty of Medicine, School of Health Sciences, University of Thessaly, \\ 41110 Biopolis, Larissa, Greece
}

Correspondence should be addressed to Dimitrios P. Bogdanos; dimitrios.bogdanos@kcl.ac.uk

Received 28 June 2013; Accepted 22 August 2013

Academic Editor: Yehuda Shoenfeld

Copyright @ 2013 Daniel S. Smyk et al. This is an open access article distributed under the Creative Commons Attribution License, which permits unrestricted use, distribution, and reproduction in any medium, provided the original work is properly cited.

Background and Aim. Neonatal hemochromatosis $(\mathrm{NH})$ is characterised by severe liver injury and extrahepatic siderosis sparing the reticuloendothelial system. Its aetiology is obscure, although it has been proposed as an alloimmune disease, resulting from immunological reaction to self-antigens (alloantigens) which the body recognizes as foreign. We studied an infant with $\mathrm{NH}$ and his mother whose sera contained antimitochondrial antibody (AMA), the hallmark of primary biliary cirrhosis (PBC). Material and Methods. To investigate the origin of AMA in the infant, we studied isotype distributions in serum from the mother and infant. Serum samples were obtained at diagnosis of NH, after liver transplantation (LT; age 1 month), and over the ensuing 17 months. Results. At NH diagnosis, infant and maternal serum contained AMA of the IgG isotype, predominantly of the G3 and G1 subclasses. AMA strongly reacted against the pyruvate dehydrogenase complex E2 subunit (PDC-E2), the major PBC-specific AMA autoantigen. Anti-PDC-E2 responses in both infant and mother declined over time, being present 2 months after LT (mother and child) and absent 10 months later (mother) and 17 months later (child). Conclusion. The association of maternally transferred IgG1 and IgG3 subclass AMA with the appearance of liver damage in an infant with NH may suggest a causal link between antibody and liver damage.

\section{Introduction}

Neonatal haemochromatosis $(\mathrm{NH})$ is a rare condition of unknown aetiology characterised by perinatal liver failure and extrahepatic siderosis sparing the reticuloendothelial system $[1,2]$. No genetic or infectious factors have been identified to explain its recurrence rate of $60-80 \%$ in pregnancies that follow an index case $[1,3-7]$. The recent suggestion that $\mathrm{NH}$ is an alloimmune disease is based on a recurrence pattern similar to that of other alloimmune diseases, on the ability of administration of intravenous immunoglobulin during pregnancy substantially to ameliorate or even to prevent fetal liver disease in siblings of an index patient, and on the demonstration that patient hepatocytes bear assembled components of the terminal complement cascade pathway together with IgG of maternal origin [2, 7-11].

A direct proof that $\mathrm{NH}$ is alloimmune is missing since no target has been identified for the putative alloantibody $[9,12]$. Autoantibodies of maternal origin, such as anti-Ro and antiLa, have been described in some children with $\mathrm{NH}[3,13-17]$. The relevance of these antibodies, which are not liver specific [18], to the pathogenic process in $\mathrm{NH}$ is unclear, although they have been associated with liver disease in rare cases [19].

We investigated an infant with $\mathrm{NH}$ whose serum contained antimitochondrial antibody (AMA), the hallmark of primary biliary cirrhosis $(\mathrm{PBC})$, a disease with a striking (>95\%) female preponderance typically affecting middleaged women $[20,21]$. We describe the characteristics of the 
AMA found in this infant, demonstrate its maternal origin, and discuss its potential relevance to the development of $\mathrm{NH}$.

\section{Material and Methods}

2.1. Subjects. Serum samples from a mother and her infant, who had NH, were obtained. This was the first pregnancy of a 27-year-old of Eastern European origin with no significant medical history complicated by oligohydramnios, symmetrical intrauterine growth retardation, and reduced fetal movements. After spontaneous labour at term she was delivered vaginally of a boy weighing $2.270 \mathrm{~kg}$ (small for gestational age; $<$ second centile) with a head circumference of $33 \mathrm{~cm}(<0.4$ centile). Apgar scores were not recorded and the placenta was not examined; the infant was described as in good condition. Left hip dislocation was noted.

Evaluation for poor feeding and hypoglycaemia at age 2 days identified jaundice with coagulopathy [INR 2.3, normal value $(\mathrm{nv})<1.2$ ], resistant to parenteral vitamin $\mathrm{K}$. Liver synthetic function was poor [serum albumin $22 \mathrm{~g} / \mathrm{L}$, normal range (nr): 35-50]. Indices of hepatic and renal function declined despite treatment for presumed sepsis and the boy was transferred to the Paediatric Liver Centre at King's College Hospital at 7 days of age. $\mathrm{NH}$ was suspected in view of the patient's age, history, and clinical-laboratory test results [serum ferritin $1481 \mathrm{ng} / \mathrm{mL}$ (nr: 20-300), INR 5.37, albumin $26 \mathrm{~g} / \mathrm{L}$, AST $44 \mathrm{IU} / \mathrm{L}$ (nr: 10-50), ALT $30 \mathrm{IU} / \mathrm{L}$ (nr: 10-50), and total bilirubin $292 \mu \mathrm{mol} / \mathrm{L}$ (nr: 3-20)]. Before blood transfusion, total iron binding capacity was $28 \mu \mathrm{mol} / \mathrm{l}$ (nr: 50-72) and transferrin saturation was 72\% (nr: 20-50\%) in peripheral-blood serum. Serum immunoglobulin concentrations were IgG, $5.53 \mathrm{~g} / \mathrm{L}$ (nr: 5-13); IgM, $0.63 \mathrm{~g} / \mathrm{L}$ (nr: $0.05-0.2$ ); and $\operatorname{IgA}, 0.63 \mathrm{~g} / \mathrm{L}$ (nr: 0.01-0.08). Autoantibody testing by indirect immunofluorescence and molecular assays revealed the presence of $\mathrm{PBC}$-specific AMA in serum from the child and his mother (see below).

The diagnosis of $\mathrm{NH}$ was confirmed at 12 days of age by microscopy of a lower lip mucosa biopsy specimen, which found haemosiderin granules within submucosal acinar gland cells (Figure 1). He was treated with intravenous desferrioxamine, sodium selenite pentahydrate, and $\mathrm{N}$-acetylcysteine and was supported with blood and blood-product transfusions. At age 10 and 16 days he was given intravenous immunoglobulin $(0.5 \mathrm{~g} / \mathrm{kg})$, and at 21 days he underwent single-volume exchange transfusion [22].

Poor clinical status prompted listing for liver transplantation (LT) at age 12 days. He received a cytomegalovirus mismatched (donor+/recipient-) split liver allograft at age 30 days. Examination of the explanted liver found postnecrotic cirrhosis with canalicular and hepatocellular cholestasis (Figure 2).

He made a slow but steady recovery. Following an isolated episode of lip smacking, and in view of microcephaly he underwent full neurological assessment, which found no cause for seizures; magnetic resonance imaging demonstrated normal brain structures with turricephaly. He was discharged home aged 92 days, 2 months after LT. Hip dislocation was successfully treated at age 10 months with reduction and casting. Allergy to milk, wheat, and egg

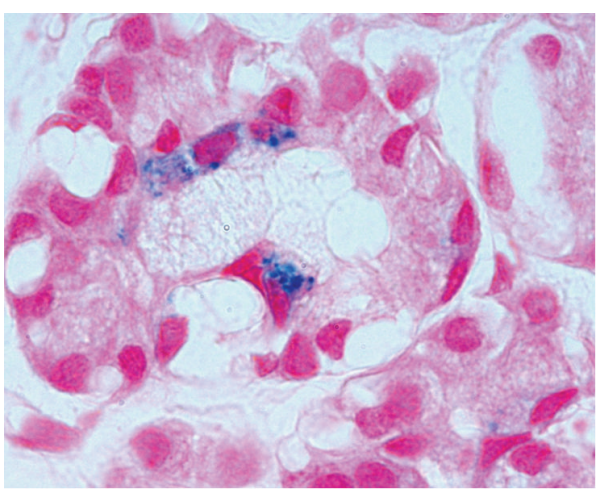

FIGURE 1: Granules of stainable iron (haemosiderin) within cytoplasm of epithelium of minor glands of the mucosa of the lower lip. Perls' technique with nuclear fast red counterstain, original magnification $1,000 x$.

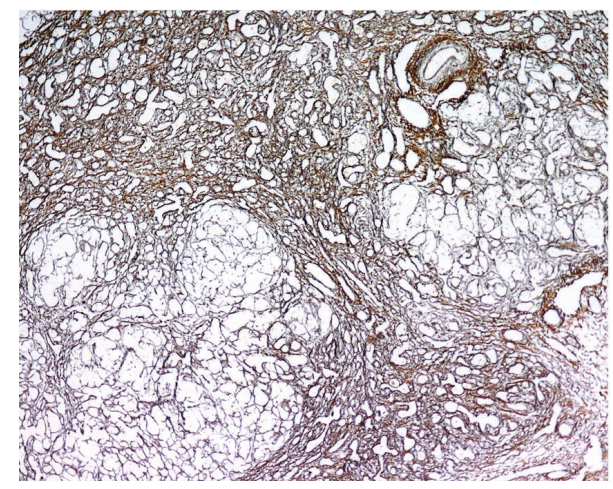

Figure 2: Hepatectomy specimen, postnatal age 30 days. Nodularity is apparent. The relatively stroma-poor nodules consisted of hepatocellular parenchyma, sometimes cholestatic. The intervening regions that exhibit more compact connective-tissue elements were sites of hepatocellular loss and stromal collapse, with neocholangiolar transformation of remaining parenchyma. Reticulin, original magnification 40x.

antigens was diagnosed at age 13 months during evaluation of an urticarial rash. Epstein-Barr virus (EBV) infection was diagnosed at age 16 months (EBV DNA 136,907 copies/mL, blood) and at age 19 months upper respiratory tract infection accompanied fever and widespread lymphoadenopathy (EBV DNA 3,653,748 copies/mL). Adjustment in immunosuppressive regimen was rapidly followed by clinical improvement. Development was normal at age 22 months, without clinicalbiochemistry evidence of hepatobiliary disease, although EBV viraemia persisted (EBV DNA 2,169,653 copies/mL).

2.2. Autoantibody Testing. Serum samples obtained from the child at presentation and over the 17 months thereafter (including samples before and after LT) and from the mother at diagnosis of $\mathrm{NH}$ in the infant and 4 and 10 months after giving birth were tested for autoantibodies. An informed consent was obtained. Autoantibody testing complied with the principles laid down in the Declaration of Helsinki. Samples were stored at $-80^{\circ} \mathrm{C}$ until use. 
Autoantibodies were sought by indirect immunofluorescence (IFL) using as substrate rat liver/kidney/stomach tissue (KSL Slide, INOVA, San Diego, CA) and human epithelial (HEp-2) cells (INOVA) at a starting serum dilution of $1 / 20$ to extinction. Autoantibodies to liver and nuclear antigens were tested by a combination of immunoassays including ELISAs (INOVA; EUROIMMUN, Lübeck, Germany), multiparametric line immunoassay (EUROIMMUN), and Western blot. These assays were based on purified M2 (AMA) antigen from porcine heart mitochondria; on individual human recombinant E2 pyruvate dehydrogenase complex (PDC), branched chain oxoacid dehydrogenase complex (BCOADC), and oxoglutarate dehydrogenase complex (OGDC) expressed in a baculovirus system (DIARECT, Freiburg, Germany); or on extracts from primate liver or from HEp-2 cell nuclei, as described [23-27].

AMA was initially detected by IFL. It was then characterised by line immunoassay and by an MIT3 ELISA whose target is a hybrid molecule comprising the major epitopes of PDC-E2, BCOADC-E2, and OGDC-E2 combined with native M2 antigen (M2-3E ELISA, EUROIMMUN). To investigate the specificity of the AMA response, reactivity against $\mathrm{PBC}$-specific mitochondrial autoantigens was tested by in house ELISAs. These used recombinant PDC-E2, BCOADC-E2, and OGDC-E2 antigens (DIARECT) as individual targets. Briefly, wells of a flat-bottom plate (MaxiSorp, NUNC, Thermo Scientific, Roskilde, Denmark) were coated in triplicate with individual PDC-E2 $(1 \mu \mathrm{g} / \mathrm{mL})$, BCOADCE2 $(1 \mu \mathrm{g} / \mathrm{mL})$, and OGDC-E2 $(2 \mu \mathrm{g} / \mathrm{mL})$ diluted in phosphate buffered saline (PBS, Sigma-Aldrich, Coylton, Ayrshire, UK) containing $0.1 \%$ bovine serum albumin (BSA) at $37^{\circ} \mathrm{C}$ for $1 \mathrm{~h}$. After a blocking step with $2 \%$ BSA for $1 \mathrm{~h}$ followed by 3 washes, serum samples (1/100) in $0.1 \% \mathrm{PBS} /$ Tween 20 (Sigma-Aldrich) were added to the coated wells and the plates were incubated for $1.5 \mathrm{~h}$ at room temperature on a shaker. After washing (3x), a horseradish peroxidase-conjugated anti-human IgG (INOVA) was added. The plates were held at room temperature for 45 minutes and the chromogenic substrate tetramethylbenzidine (INOVA) was added. The plates were then kept at room temperature for $15 \mathrm{~min}$ in the dark. Reaction was terminated with $100 \mu \mathrm{L} /$ well of $4 \mathrm{~N}$ $\mathrm{H}_{2} \mathrm{SO}_{4}$ and absorbance (optical density, OD) at $450 \mathrm{~nm}$ was read against blank wells in a microplate reader (MRX, Dynex Technologies).

IgG subclass-specific anti-PDC-E2 reactivity was investigated by in-house ELISAs, using horseradish peroxidaselabelled anti-IgG1, IgG2, IgG3, and IgG4 (SouthernBiotech, Birmingham, AL), as described [28]. Each sample was tested in triplicate and the mean optical density was calculated.

\section{Results}

At time of presentation to our unit on day 7 of life, the infant's serum contained AMA of the IgG isotype at a titre of $1: 80$ by IFL; the mother's serum obtained at the same time contained AMA at a titre of $1: 320$ for IgG and $1: 40$ for IgM. Other autoantibodies, including anti-nuclear, antismooth muscle, anti-liver kidney microsomal, anti-double stranded DNA, and anti-extractable nuclear antigens, could not be demonstrated in either.

The AMA of infant and mother reacted only with PDCE2 (Figure 3(a)) and belonged to the IgG class. The infant autoantibody belonged predominantly to the IgG3 and to a lesser extent to the IgG1 isotype (Figure 3(b)). There was no anti-PDC-E2 reactivity of the IgM or IgA isotype. A similar anti-PDC-E2 antibody distribution was seen in the mother, with the difference that IgG4 and IgM $(1: 40)$ anti-PDC-E2 antibodies also were present (Figure 3(b)). Concentrations of IgG2 anti-PDC-E2 antibodies were negligible in both mother and infant. At age 4 months (3 months after LT), the child's serum still reacted against PDC-E2, although the antibody titre was lower than that at diagnosis (Figure 3(c)). Anti-PDC-E2 reactivity also declined in the mother's serum (Figure 3(c)). Anti-PDC-E2 antibodies were borderline demonstrable in the child at age 10 months and were undetectable in contemporaneously obtained serum from his mother. At age 17 months, anti-PDC-E2 reactivity in the child had declined below the threshold of detectability (OD: 0.2; cut off: 0.24; Figure 3(c)). IgM- or IgA-class anti-PDC-E2 antibodies were never observed in the child.

\section{Discussion}

AMA, a liver-disease-specific autoantibody, is reported here for the first time in an infant with $\mathrm{NH}$. All the evidence indicates that his AMA was of maternal origin, since it belonged to the placenta-crossing IgG class and showed a similar IgG3 and IgG1 pattern in mother and baby $[29,30]$. The mother's serum also contained small quantities of AMA of IgG4 and IgM isotypes, which do not cross the placenta [29]. Both in mother and baby, AMA targeted PDC-E2, the major AMA antigen in $\mathrm{PBC}$, but was peculiarly unreactive with BCOADC-E2 and OGDC-E2, which in PBC are usually recognised by sera that contain anti-PDC-E2 antibodies [20, 21,31 .

Whitington and colleagues described in vitro binding to hepatocytes by maternal antibodies of the IgG1 and IgG3 isotypes, thought to target liver alloantigens in infants with $\mathrm{NH}$ after crossing the placenta [8,9]. IgGs isolated from these infants are able to bind to murine hepatocytes and to induce their necrosis through complement activation $[8,9]$, an event immunohistochemically reflected in the assembly of the terminal complement components on the hepatocytes of NH infants [10].

The AMA present in our mother-baby pair targets PDC$\mathrm{E} 2$, an autoantigen typically recognised by the AMA of $\mathrm{PBC}$ patients, and belongs to the complement-fixing IgG3 and IgG1 isotypes, akin to the antibodies described by Whitington [8]. IgG3 and IgG1 antibodies are particularly efficient at activating the complement system and at recruiting cells bearing the $\mathrm{Fc}$ receptors that are capable of phagocytosis and antibody-dependent cell-mediated cytotoxicity [32-34]. Of note is that the ability of AMA to activate the complement system has been described in patients with $\mathrm{PBC}[35,36]$.

If the IgG3/IgG1 AMA present in our infant recognised a pathogenetically relevant liver target, as proposed for $\mathrm{PBC}$ 

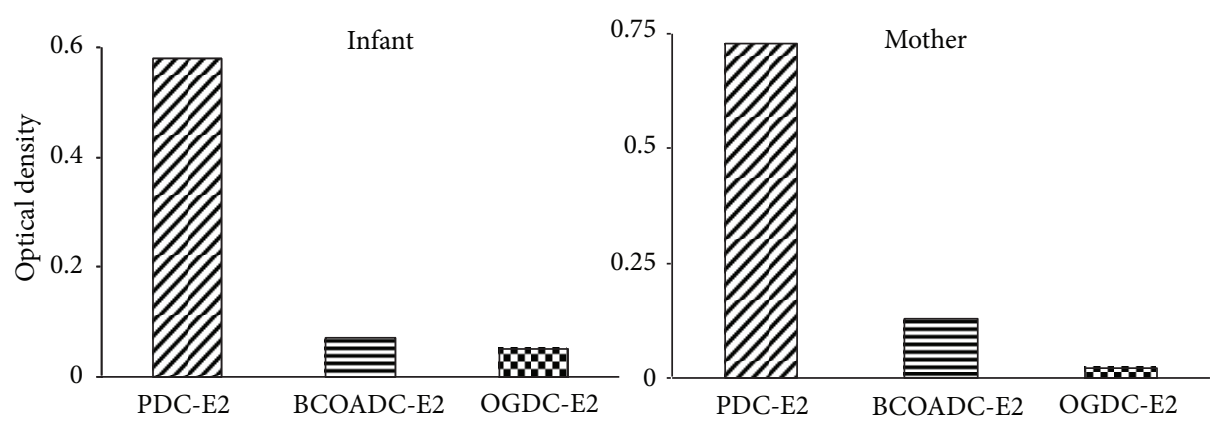

\section{$\mathbb{Z}$ PDC-E2 ECOADC-E2}

B OGDC-E2

(a) The AMAs present in these samples are predominantly directed against the E2 subunit of pyruvate dehydrogenase complex (PDC-E2), which is the major AMA autoantigen in patients with primary biliary cirrhosis. Antibody reactivity to the E2 subunits of branched chain oxoacid dehydrogenase complex (BCOADC-E2) and oxoacid dehydrogenase complex (OGDC-E2), which are also immunodominant antigens, is not detectable. The dashed line represents the cut-off of 0.24 (mean average +5 standard deviations) of 28 normal controls tested in triplicate

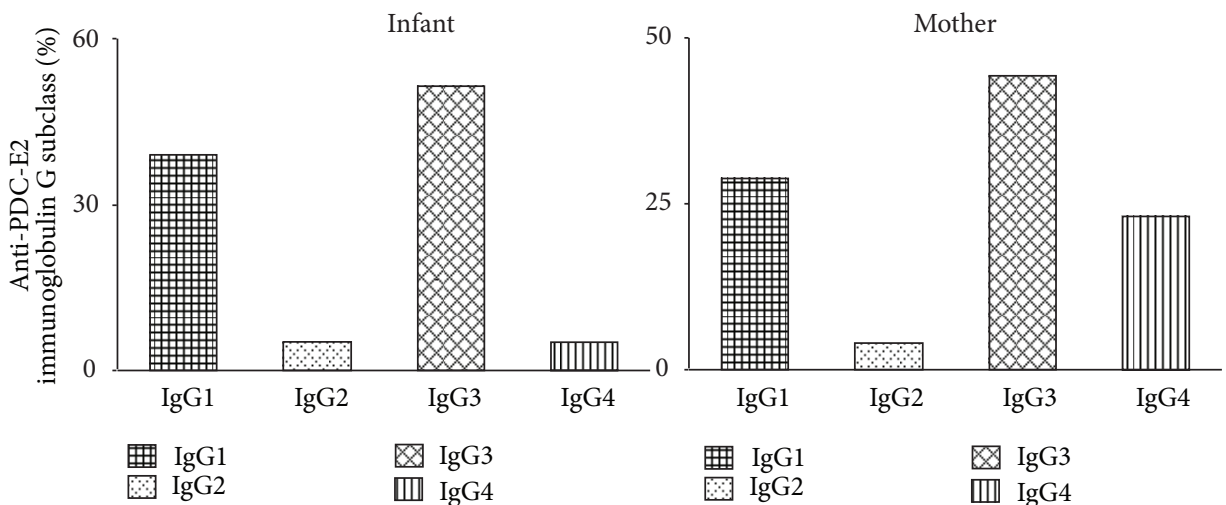

(b) Anti-PDC-E2 IgG subclasses are similarly distributed in the infant and his mother, with predominance of IgG3 and IgG1. The mother's serum also contains anti-PDC-E2 of IgG4 type, which does not cross the placenta

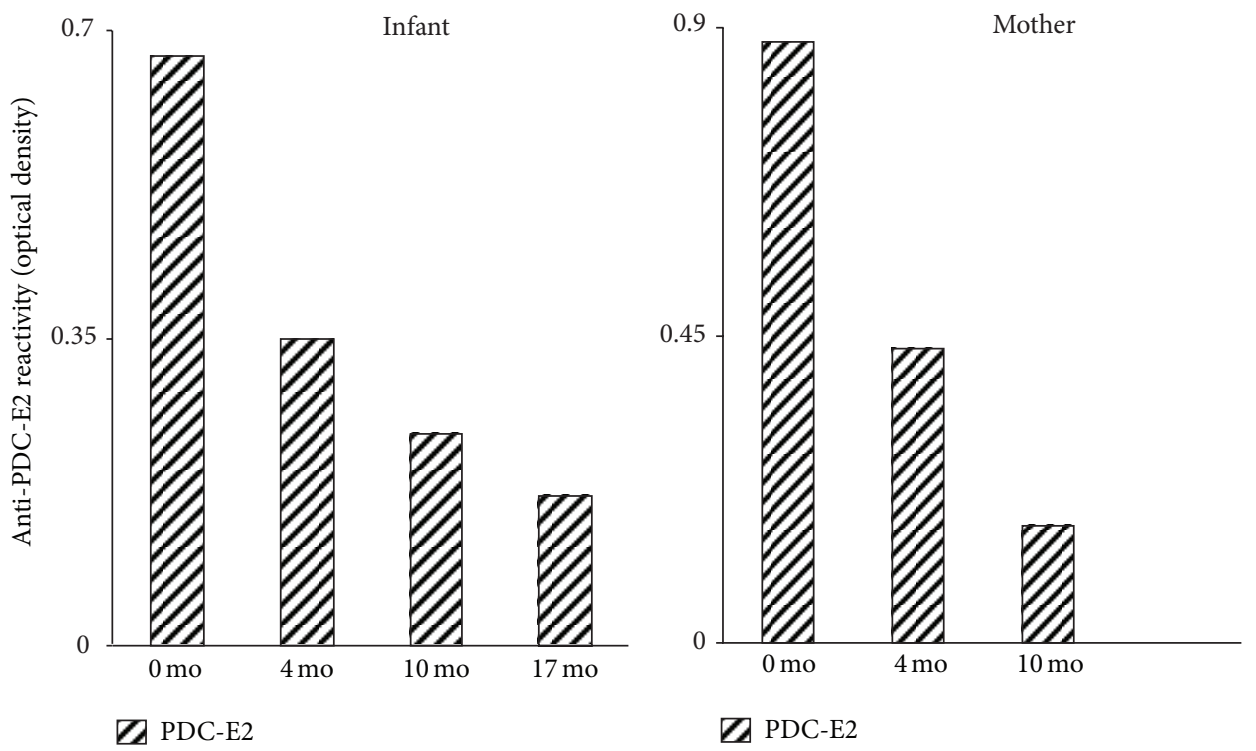

(c) Anti-PDC-E2 antibodies declined in titre over time and were well below the cut-off value in both mother and infant 10 months after delivery

FIGURE 3: Antigen specific anti-mitochondrial antibody (AMA) reactivity in the infant and his mother tested by ELISA. Optical density $(y$-axis) and time elapsed since birth of child ( $x$-axis) are indicated. 
$[37,38]$, it may have contributed to the development of severe liver damage and failure.

AMA has been occasionally described in liver disease in paediatric patients. Gregorio et al. reported a girl who at age 12 years developed autoimmune hepatitis with $\mathrm{PBC}$ specific AMA [39]. AMA persisted throughout the course of her disease despite treatment; the patient died at age 24 years with liver failure, following several episodes of nonadherence to treatment and spontaneous bacterial peritonitis [39]. More pertinent to our case, placentally transferred PDCE2-targeting IgG3 and IgG1 AMAs have been reported in 2 infants with acute liver injury that lasted until the antibody disappeared [28].

The AMA present in the mother of our patient with $\mathrm{NH}$ is of interest for two reasons: it partially belonged to the IgM isotype, suggesting that it was produced as part of a primary autoimmune response; it declined in concentration over time and eventually disappeared, implying that the triggering autoantigenic stimulus had subsided. A loss of AMA, though occasionally reported, is unusual, since AMA tends to persist once detected, heralding the development of $\mathrm{PBC}$ [40]. The mother of the infant reported here is regularly monitored for a possible reappearance of AMA, especially in the case of a new pregnancy.

Recent reports show that AMA can be detected in some $40 \%$ of patients with acute liver failure when the antibody is sought with a highly sensitive MIT3 ELISA [41, 42]. In our study an MIT3 ELISA was used to confirm IFL results; it demonstrated AMA in both the infant and his mother. Akin to the AMA characterised in the present study, the AMAs described by Leung et al. using MIT3 ELISA were transient [41]. Their disappearance was attributed to lack of genetic susceptibility to PBC. The real prevalence and possible involvement of AMA in the causation of the severe liver damage accompanying $\mathrm{NH}$ will need to be investigated using similarly sensitive techniques in a series of affected infants.

Although we cannot definitively link AMA with liver injury in this infant, and $\mathrm{NH}$ is not a known complication of pregnancy in women with $\mathrm{PBC}$, no other cause of liver injury was identified. We also cannot rule out AMA as being associated with the liver disease seen in this infant, as the transplacental passage of AMA has been reported in infants with liver injury of unknown cause [28]. As well, the prevalence of AMA in NH patients and/or their mothers has not been evaluated.

\section{List of Abbreviations}

AMA:

Antimitochondrial antibodies

ALT:

Alanine transaminase

AST:

Aspartate aminotransferase

BCOADC-E2: Branched-chain oxoacid dehydrogenase complex E2 subunit

EBV: $\quad$ Epstein-Barr virus

ELISA: $\quad$ Enzyme linked immunosorbent assay

IgG/A/M: Immunoglobulin G/A/M

IFL: Indirect immunofluorescence

INR: International Normalised Ratio

LT: $\quad$ Liver transplantation
NH: $\quad$ Neonatal haemochromatosis

OGDC-E2: Oxoglutarate dehydrogenase complex E2 subunit

OD: $\quad$ Optical density

PBC: $\quad$ Primary biliary cirrhosis

PDC-E2: Pyruvate dehydrogenase complex E2 subunit

PBS: $\quad$ Phosphate buffered saline.

\section{Conflict of Interests}

The authors declare that they have no competing interests.

\section{Authors' Contributions}

Daniel S. Smyk clerked the infant and mother as a medical student in paediatrics, wrote the first draft and contributed to and revised subsequent drafts of the manuscript; Maria G. Mytilinaiou conducted the immunological analysis; Tassos Grammatikopoulos was involved in the clinical management of the patient, and contributed to the writing of the manuscript; A. S. Knisely conducted the histopathological analysis, provided histological images, and contributed to writing and revising the manuscript; Giorgina Mieli-Vergani was involved in the clinical management of the patient and contributed to the writing and revising of the manuscript; Diego Vergani contributed to the writing and revising of the manuscript; Dimitrios P. Bogdanos had the overall supervision of the study, conducted the immunological analysis, was involved in the clinical evaluation of the mother, and contributed to the writing and revising of the manuscript.

\section{References}

[1] A. S. Knisely, G. Mieli-Vergani, and P. F. Whitington, "Neonatal hemochromatosis," Gastroenterology Clinics of North America, vol. 32, no. 3, pp. 877-889, 2003.

[2] P. F. Whitington, "Gestational alloimmune liver disease and neonatal hemochromatosis," Seminars in Liver Disease, vol. 32, no. 4, pp. 325-332, 2012.

[3] A. L. Kelly, P. W. Lunt, F. Rodrigues et al., "Classification and genetic features of neonatal haemochromatosis: a study of 27 affected pedigrees and molecular analysis of genes implicated in iron metabolism," Journal of Medical Genetics, vol. 38, no. 9, pp. 599-610, 2001.

[4] T. M. Cox and D. J. Halsall, "Hemochromatosis-neonatal and young subjects," Blood Cells, Molecules \& Diseases, vol. 29, no. 3, pp. 411-417, 2002.

[5] J. Hoogstraten, D. J. de Sa, and A. S. Knisely, "Fetal liver disease may precede extrahepatic siderosis in neonatal hemochromatosis," Gastroenterology, vol. 98, no. 6, pp. 1699-1701, 1990.

[6] M. M. Kershisnik, A. S. Knisely, C.-C. J. Sun, J. M. Andrews, and C. T. Wittwer, "Cytomegalovirus infection, fetal liver disease, and neonatal hemochromatosis," Human Pathology, vol. 23, no. 9, pp. 1075-1080, 1992.

[7] P. F. Whitington and J. U. Hibbard, "High-dose immunoglobulin during pregnancy for recurrent neonatal haemochromatosis," The Lancet, vol. 364, no. 9446, pp. 1690-1698, 2004. 
[8] P. F. Whitington, "Neonatal hemochromatosis: a congenital alloimmune hepatitis," Seminars in Liver Disease, vol. 27, no. 3, pp. 243-250, 2007.

[9] P. F. Whitington and P. Malladi, "Neonatal hemochromatosis: is it an alloimmune disease?" Journal of Pediatric Gastroenterology and Nutrition, vol. 40, no. 5, pp. 544-549, 2005.

[10] X. Pan, S. Kelly, H. Melin-Aldana, P. Malladi, and P. F. Whitington, "Novel mechanism of fetal hepatocyte injury in congenital alloimmune hepatitis involves the terminal complement cascade," Hepatology, vol. 51, no. 6, pp. 2061-2068, 2010.

[11] S. Bonilla, J. D. Prozialeck, P. Malladi et al., "Neonatal iron overload and tissue siderosis due to gestational alloimmune liver disease," Journal of Hepatology, vol. 56, no. 6, pp. 1351-1355, 2012.

[12] A. S. Knisely and D. Vergani, "Neonatal hemochromatosis: a revision," Hepatology, vol. 51, no. 6, pp. 1888-1890, 2010.

[13] G. Timpani, F. Foti, A. Nicolò, P. A. Nicotina, E. Nicastro, and R. Iorio, "Is exchange transfusion a possible treatment for neonatal hemochromatosis?" Journal of Hepatology, vol. 47, no. 5, pp. 732-735, 2007.

[14] F. Rodrigues, M. Kallas, R. Nash et al., "Neonatal hemochromatosis-medical treatment vs. transplantation: the King's experience," Liver Transplantation, vol. 11, no. 11, pp. 1417-1424, 2005.

[15] D. M. Flynn, N. Mohan, P. McKiernan et al., "Progress in treatment and outcome for children with neonatal haemochromatosis," Archives of Disease in Childhood, vol. 88, no. 2, pp. F124F127, 2003.

[16] J. Schoenlebe, J. P. Buyon, B. J. Zitelli, D. Friedman, M. A. Greco, and A. S. Knisely, "Neonatal hemochromatosis associated with maternal autoantibodies against Ro/SS-A and La/SS-B ribonucleoproteins," American Journal of Diseases of Children, vol. 147, no. 10, pp. 1072-1075, 1993.

[17] S. Collardeau-Frachon, S. Heissat, R. Bouvier et al., "French retrospective multicentric study of neonatal hemochromatosis: importance of autopsy and autoimmune maternal manifestations," Pediatric and Developmental Pathology, vol. 15, no. 6, pp. 450-470, 2012.

[18] C. Defendenti, F. Atzeni, M. F. Spina et al., "Clinical and laboratory aspects of Ro/SSA-52 autoantibodies," Autoimmunity Reviews, vol. 10, no. 3, pp. 150-154, 2011.

[19] S.-C. Lin, S.-D. Shyur, L.-H. Huang, J.-Y. Wu, H.-T. Chuo, and H.-C. Lee, "Neonatal lupus erythematosus with cholestatic hepatitis," Journal of Microbiology, Immunology and Infection, vol. 37, no. 2, pp. 131-134, 2004.

[20] M. M. Kaplan and M. E. Gershwin, "Primary biliary cirrhosis," The New England Journal of Medicine, vol. 353, no. 12, pp. 12611273, 2005.

[21] D.-P. Bogdanos, H. Baum, and D. Vergani, "Antimitochondrial and other autoantibodies," Clinics in Liver Disease, vol. 7, no. 4, pp. 759-777, 2003.

[22] E. B. Rand, S. J. Karpen, S. Kelly et al., "Treatment of neonatal hemochromatosis with exchange transfusion and intravenous immunoglobulin," Journal of Pediatrics, vol. 155, no. 4, pp. 566.el-571.el, 2009.

[23] D. P. Bogdanos and L. Komorowski, "Disease-specific autoantibodies in primary biliary cirrhosis," Clinica Chimica Acta, vol. 412, no. 7-8, pp. 502-512, 2011.

[24] H. Liu, G. L. Norman, Z. Shums et al., "PBC screen: an IgG/IgA dual isotype ELISA detecting multiple mitochondrial and nuclear autoantibodies specific for primary biliary cirrhosis," Journal of Autoimmunity, vol. 35, no. 4, pp. 436-442, 2010.
[25] E. I. Rigopoulou, E. T. Davies, D.-P. Bogdanos et al., "Antimitochondrial antibodies of immunoglobulin G3 subclass are associated with a more severe disease course in primary biliary cirrhosis," Liver International, vol. 27, no. 9, pp. 1226-1231, 2007.

[26] E. I. Rigopoulou, D.-P. Bogdanos, C. Liaskos et al., "Anti-mitochondrial antibody immunofluorescent titres correlate with the number and intensity of immunoblot-detected mitochondrial bands in patients with primary biliary cirrhosis," Clinica Chimica Acta, vol. 380, no. 1-2, pp. 118-121, 2007.

[27] C. Dähnrich, A. Pares, L. Caballeria et al., "New ELISA for detecting primary biliary cirrhosis-specific antimitochondrial antibodies," Clinical Chemistry, vol. 55, no. 5, pp. 978-985, 2009.

[28] S. Hannam, D.-P. Bogdanos, E. T. Davies et al., "Neonatal liver disease associated with placental transfer of anti-mitochondrial antibodies," Autoimmunity, vol. 35, no. 8, pp. 545-550, 2002.

[29] F. Saji, Y. Samejima, S. Kamiura, and M. Koyama, "Dynamics of immunoglobulins at the feto-maternal interface," Reviews of Reproduction, vol. 4, no. 2, pp. 81-89, 1999.

[30] N. E. Simister, "Placental transport of immunoglobulin G," Vaccine, vol. 21, no. 24, pp. 3365-3369, 2003.

[31] D. P. Bogdanos, P. Invernizzi, I. R. Mackay, and D. Vergani, "Autoimmune liver serology: current diagnostic and clinical challenges," World Journal of Gastroenterology, vol. 14, no. 21, pp. 3374-3387, 2008.

[32] M. Bruggemann, G. T. Williams, C. I. Bindon et al., "Comparison of the effector functions of human immunoglobulins using a matched set of chimeric antibodies," The Journal of Experimental Medicine, vol. 166, no. 5, pp. 1351-1361, 1987.

[33] V. N. Schumaker, M. A. Calcott, H. L. Spiegelberg, and H. J. Müller-Eberhard, "Ultracentrifuge studies of the binding of IgG of different subclasses to the Clq subunit of the first component of complement," Biochemistry, vol. 15, no. 23, pp. 5175-5181, 1976.

[34] M. R. Clark, "IgG effector mechanisms," Chemical Immunology, vol. 65, pp. 88-110, 1997.

[35] J. R. Wands, J. L. Dienstag, A. K. Bhan, E. R. Feller, and K. J. Isselbacher, "Circulating immune complexes and complement activation in primary biliary cirrhosis," The New England Journal of Medicine, vol. 298, no. 5, pp. 233-237, 1978.

[36] P. A. Berg, D. Doniach, and I. M. Roitt, "Mitochondrial antibodies in primary biliary cirrhosis. I. Localization of the antigen to mitochondrial membranes," The Journal of Experimental Medicine, vol. 126, no. 2, pp. 277-290, 1967.

[37] G. Rong, R. Zhong, A. Lleo et al., "Epithelial cell specificity and apotope recognition by serum autoantibodies in primary biliary cirrhosis," Hepatology, vol. 54, no. 1, pp. 196-203, 2011.

[38] K. Tsuneyama, J. Van de Water, P. S. C. Leung et al., "Abnormal expression of the $\mathrm{E} 2$ component of the pyruvate dehydrogenase complex on the luminal surface of biliary epithelium occurs before major histocompatibility complex class II and BB1/B7 expression," Hepatology, vol. 21, no. 4, pp. 1031-1037, 1995.

[39] G. V. Gregorio, B. Portmann, A. P. Mowat, D. Vergani, and G. Mieli-Vergani, "A 12-year-old girl with antimitochondrial antibody-positive autoimmune hepatitis," Journal of Hepatology, vol. 27, no. 4, pp. 751-754, 1997.

[40] M. D. Van Norstrand, M. Malinchoc, K. D. Lindor et al., "Quantitative measurement of autoantibodies to recombinant mitochondrial antigens in patients with primary biliary cirrhosis: relationship of levels of autoantibodies to disease progression," Hepatology, vol. 25, no. 1, pp. 6-11, 1997. 
[41] P. S. C. Leung, L. Rossaro, P. A. Davis et al., "Antimitochondrial antibodies in acute liver failure: implications for primary biliary cirrhosis," Hepatology, vol. 46, no. 5, pp. 1436-1442, 2007.

[42] W. Bernal, F. Meda, Y. Ma, D. P. Bogdanos, and D. Vergani, "Disease-specific autoantibodies in patients with acute liver failure: the King's College London experience," Hepatology, vol. 47, no. 3, pp. 1096-1097, 2008. 


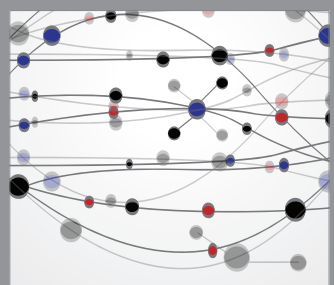

The Scientific World Journal
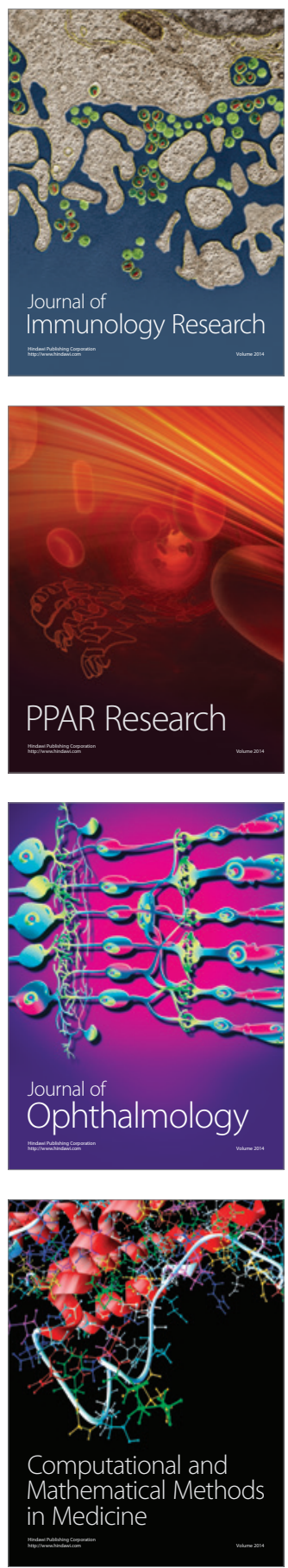

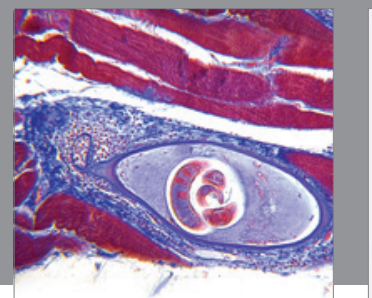

Gastroenterology

Research and Practice
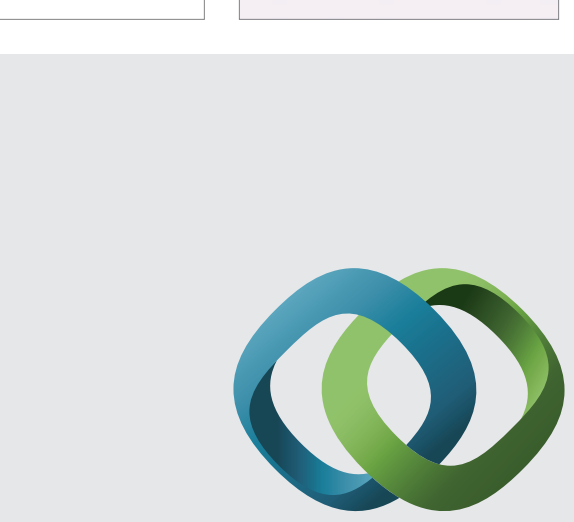

\section{Hindawi}

Submit your manuscripts at

http://www.hindawi.com
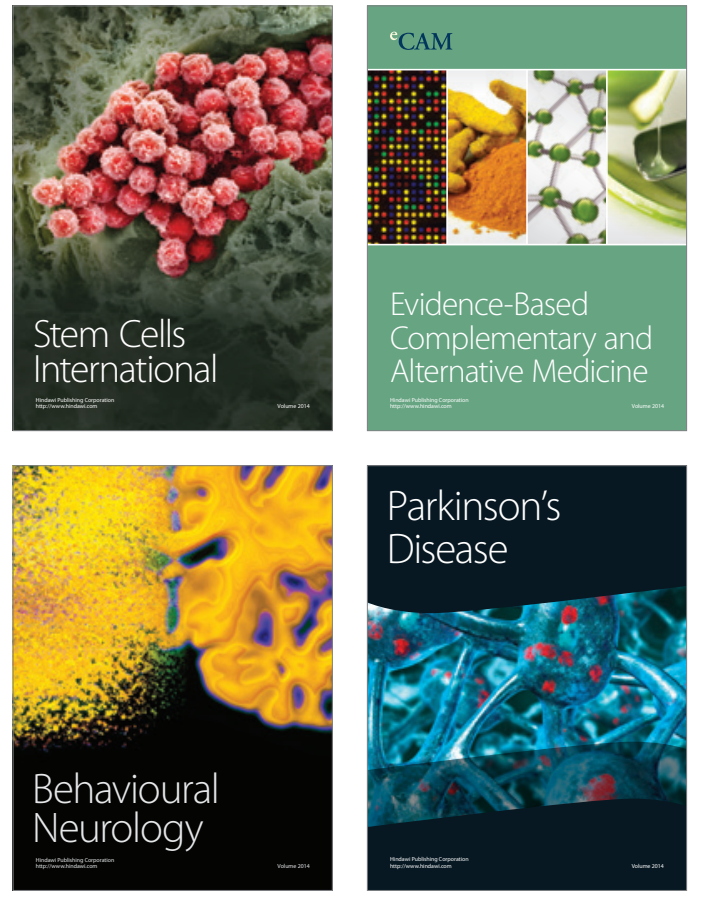
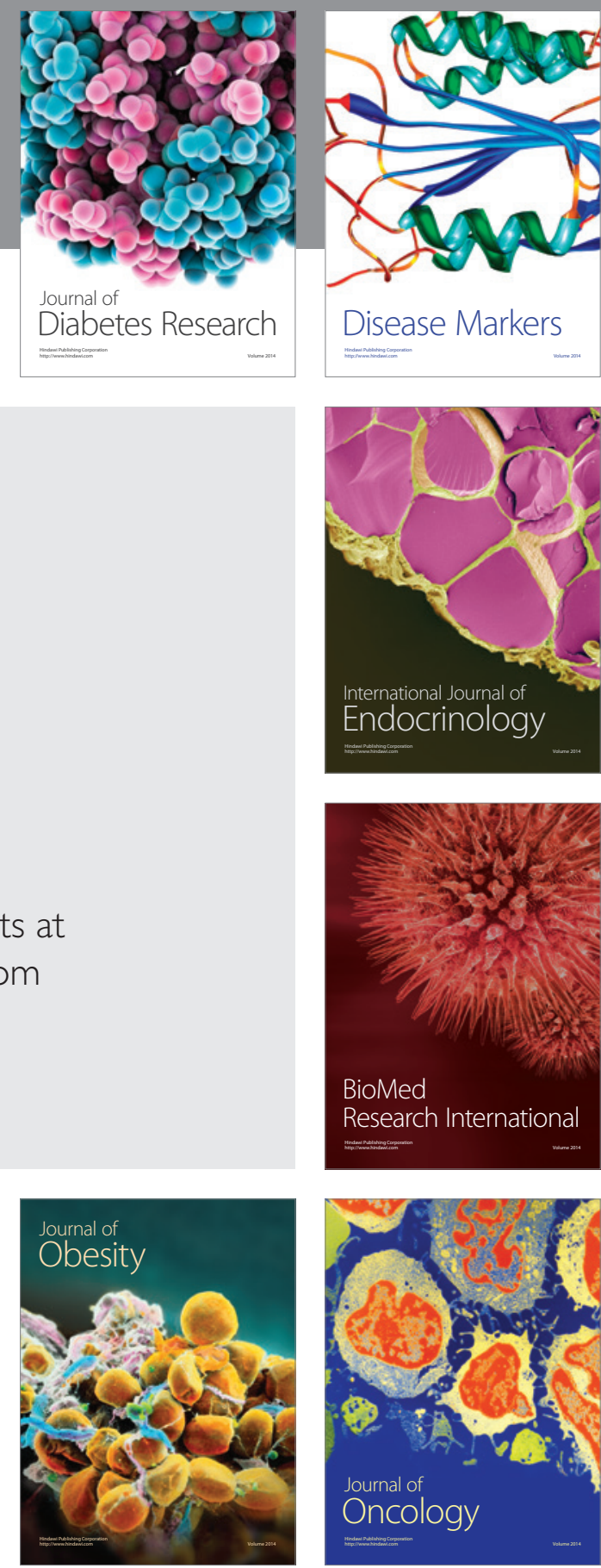

Disease Markers
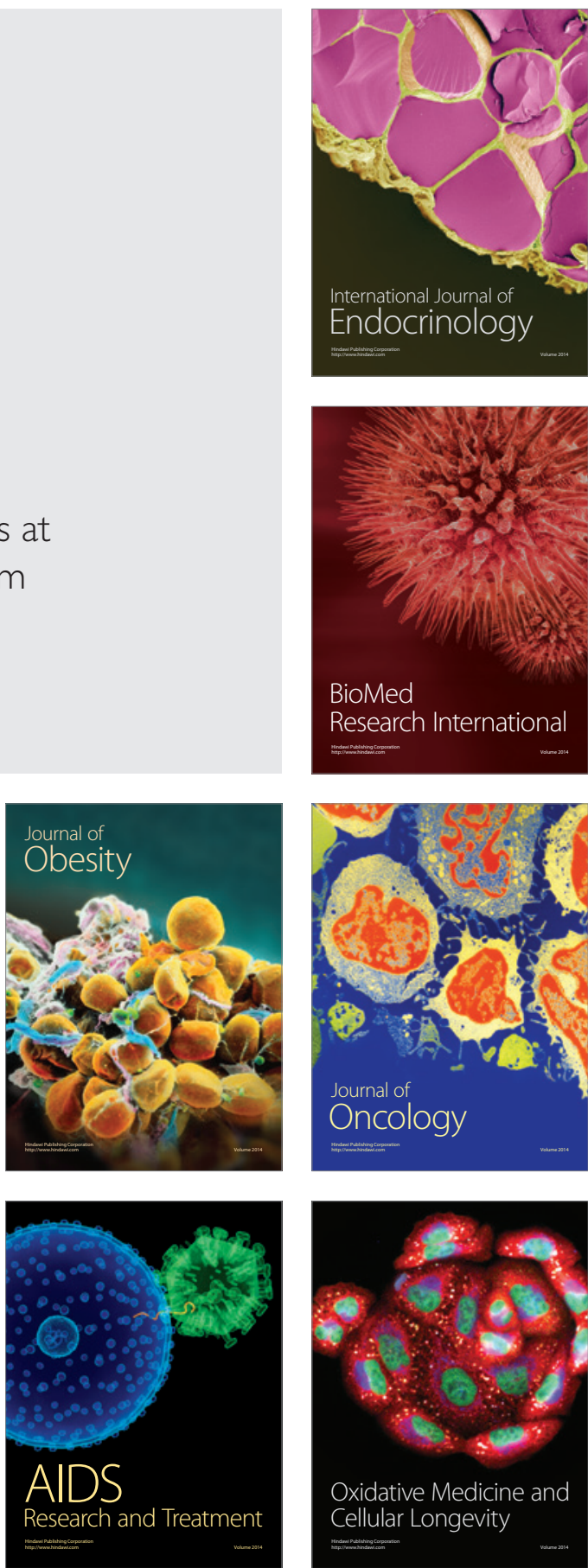